\title{
Several Jamming Attacks in Wireless Networks: A Game Theory Approach
}

\author{
Moulay Abdellatif Lmater ${ }^{1}$, Majed Haddad ${ }^{2}$, Abdelillah Karouit ${ }^{3}$ and Abdelkrim Haqiq ${ }^{4}$ \\ ${ }^{1,4}$ Networks, Mobility and Modeling laboratory, FST, Hassan 1st University, Settat, Morocco \\ ${ }^{2}$ LIA/CERI University of Avignon Agroparc, BP 1228, 84911, Avignon, France \\ ${ }^{3}$ UTEC CCI77 France
}

\begin{abstract}
Wireless jamming attacks have recently been a subject of several researches, due to the exposed nature of the wireless medium. This paper studies the anti-jamming resistance in the presence of several attackers. Two kind of jammers are considered, smart jammers which have the ability to sense the legitimate signal power and regular jammers which don't have this ability. An Anti Multi-Jamming based Power Control problem modeled as a non-zero-sum Game is suggested to study how the transmitter can adjust its signal power against several jamming attacks. A closed-form expression of Nash Equilibrium is derived when players actions are taken simultaneously. In addition, a Stackelberg Equilibrium closed-form expression is derived when the hierarchical behavior between the transmitter and jammers is assumed. Simulation results show that the proposed scheme can enhance the anti-jamming-resistance against several attackers. Furthermore, this study proves that on the transmitter side, the most dangerous jammer is considered to have the highest ratio between channel gain and jamming cost. Finally, based on the Q-Learning technique, the transmitter can learn autonomously without knowing the patterns of attackers.
\end{abstract}

Keywords-Wireless communications; game theory; jamming attacks; stackelberg game; nash game

\section{INTRODUCTION}

The massive use of wireless approaches has led to the proliferation of a multitude of new services that are becoming increasingly important for everybody. On the other hand, communication latency and energy-efficiency in the next generation networks [1], [2], [3], [4], being on the top of the increasing number of security critical services [5], are the main challenges that force telecommunication community to seek ways to enhance the wireless networks performance and reduce the risk of malicious attacks. Indeed, wireless communications are highly susceptible to jamming problems [6], [7], [8], [9] because of the exposed nature of the broadcast medium. This is the case of a large number of wireless systems based on Wireless Random Access (WRA) mechanism (for example, the 802.11 and 802.16 standards [10], [11], [12]) such as Aloha [13], Carrier Sense Multiple Access (CSMA) and their corresponding.

Jamming in wireless networks is defined as a disruption of existing wireless transmissions at various communication layers. This kind of attacks usually aims the physical layer and can be achieved by decreasing the Signal-to-Interference-plusNoise-Ratio (SINR) through the transmission of high power noise at the right moment (time slot), frequency (sub-carriers) and location (close to the transmitter or the receiver). Two kind of jammers are considered, regular jammers that are not able to sense the legitimate signal power and smart jammers that operate in jamming when they sense a transmission on the channel and has the ability to learn the ongoing signal powers, hence, this kind of jammer can adjust its own transmission power to lengthen its battery life. Initially, smart jammer keeps monitoring wireless medium in order to determine the operational frequency band on which both sides communicate. Then, it transmits a signal using that frequency band in order to reduce the SINR to a certain threshold. If the medium is in an idle state, it remains in sleep and keeps sensing the medium. Whenever a transmission fails, the transmitter doubles the back-off period and tries again, continuing with exponential back-off until the frame is successfully transmitted or the maximum number of re-transmissions is reached; the frame is then dropped and regenerated again. Consequently, jamming attacks could increase communication latency, reduce energy-efficiency and may even increase the risk of Denial-ofServices (DoS).

While measurement methods are unable to address the real scenarios and requirements due to wireless networks complexity that gives rise to time consumption during simulation process, Game Theory is an appropriate tool that would better deal with the jamming problem. In order to investigate the impact of the several jammers presence on the transmitter behavior, this paper considers the battle between the transmitter and several jammers within a single sub-carrier; the case of multi sub-carriers will be addressed in future research. This battle is modeled as an Anti Multi-Jamming based Power Control game model (AMJPC), where the transmission power is defined as a strategy of players. Since the battery life of wireless devices is directly related to the transmission activity, the players payoffs are assumed to be functions of the SINR and the transmission costs. A closed-form expression of both Nash Equilibrium (NE) and Stackelberg Equilibrium (SE) is derived. Numerical results not only describe the impact of channel gains on players utilities but also show that the jammer with the highest ratio between channel gain and jamming cost plays the role of an active player, whereas, the other ones remain inactive ; this ratio is named: the Jamming Efficiency Ratio $(\mathcal{J E R})$. Consequently, the most dangerous jammer for the transmitter is proved to have the highest $\mathcal{J E R}$. Since jamming patterns may be unknown during the battle, the worst scenario will be considered (i.e. the transmitter has partial information while the jammers have full information) so that 
the transmitter can act autonomously without knowing neither the jamming patterns and parameters nor the above game model.

The rest of the paper is organized as follows: Section II discusses some related works. Section III presents the strategic Game model. Section IV, analyzes the jamming problem according to two scenarios: 1-The presence of several regular jammers. 2-The presence of several smart jammers. Numerical results are provided in Section V. Finally, Section VI concludes the paper.

\section{RELATED WORK}

By using Game Theory formulations, previous researches on anti-jamming methods have been proposed [14], [15]. In [14], Altman et al. has employed a zero sum Game to study jamming attack in wireless networks and has assumed that the signal power can be chosen from a discrete set of power levels. In [15], authors consider a non-zero Game where the transmission cost for both jammer and transmitter is introduced. They proved the existence and uniqueness of NE. In [16], [17], [18], [19], authors assume the presence of a smart jammer and consider a hierarchical behavior between the transmitter and the jammer. This anti-jamming scenario is modeled as a Stackelberg Game. In [20], authors focus on a single jammer which keeps track of the re-transmission attempts until the packet is dropped. An anti-jamming Bayesian Stackelberg Game with incomplete information is proposed in [21]. In all previous works on anti-jamming, authors consider the battle "one transmitter - one jammer" while little attention was paid to the case of several jammers.

However, the same team in [22], extended the work in [15] to the case with several jammers modeled under a zerosum Game. they studied the Nash Equilibrium in case of regular jamming attacks. In [23], authors investigate the antijamming problem in presence of several jammers with discrete power strategies by proposing a hierarchical power control algorithm (HPCA). This paper, assumes that the power level set is continuous and proposes an AMJPC problem as a means to countermeasure jamming attacks according to two scenarios: 1) the presence of several regular jammers, 2) the presence of several smart jammers. Finally, the AMJPC model is validated based on the Q-Learning technique developed in [20].

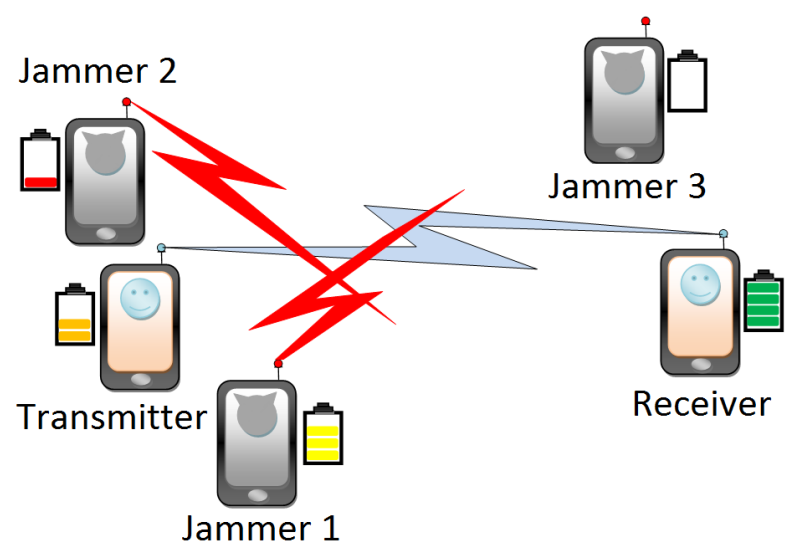

Fig. 1. Jamming Attacks

\section{SYSTEM MODEL}

Let's consider a wireless network, in which a transmitter node broadcasts legitimate signals to the receiver side. Assume that the transmitter transmits its signal in the presence of several jammers (Fig. 1), the legitimate user (the transmitter) and jammers can freely control their signal powers so as to maximize their payoffs.

Let $P \geq 0$ and $C>0$ denote the signal power and the transmission cost of the transmitter, respectively.

Similarly, let $J_{i} \geq 0$ and $E_{i}>0$ denote the signal power and the transmission cost of the jammer $i$, respectively. Hence, the SINR is formulated as follows:

$$
S I N R=\frac{\alpha P}{N+\sum_{i} \beta_{i} J_{i}}
$$

where $N$ denotes the background noise level, $\alpha>0$ and $\beta_{i}>0$ are the fading channel gains of the transmitter and the jammer $i$, respectively.

On the transmitter side, the aim is to maximize the SINR with the minimum cost, hence, based on the SINR formulation, the transmitter payoff denoted as $U$ is given by:

$$
U=\frac{\alpha P}{N+\sum_{i} \beta_{i} J_{i}}-C P
$$

However, on the jammer side, any gain of the transmitter results in its own corresponding loss. In addition, any jamming attempt from the jammer results in its own corresponding loss. As result, the jammer $i$ payoff denoted by $V_{i}$ is formulated as follows:

$$
V_{i}=-\frac{\alpha P}{N+\beta_{i} J_{i}+\sum_{j \neq i} \beta_{j} J_{j}}-E_{i} J_{i}
$$

Let's introduce a Jamming Efficiency Ratio $\left(\mathcal{J E R} \mathcal{R}_{i}\right)$ indicator that helps us to evaluate the efficiency of a jammer $J i$; which is defined by the ratio between channel gain and jamming cost, namely:

$$
\mathcal{J E} \mathcal{R}_{i}=\frac{\beta_{i}}{E_{i}}
$$

Let's consider a regular jammer and a smart jammer, where the smart one can quickly learn the transmitter's transmission power and adjust its own one accordingly to maximize its utility $V_{i}$, while the regular one doesn't have this intelligence. The aim is to determine the transmitter transmission power that maximizes the utility function $U$ and to investigate the interaction between jammers at NE/SE.

Let's now model this AMJPC scheme as a strategic Game denoted as :

$G_{N+1}=\left(\left\{\mathcal{T}, \mathcal{J}_{1}, \ldots, \mathcal{J}_{N}\right\},\left\{P, J_{1}, \ldots, J_{N}\right\},\left\{U, V_{1}, \ldots, V_{N}\right\}\right)$. In this Game, both the transmitter $(\mathcal{T})$ and jammers $\left(\mathcal{J}_{1}, \ldots, \mathcal{J}_{N}\right)$ are players. The strategies of these players are their own transmission power $\left\{P, J_{1}, \ldots, J_{N}\right\}$. Each player chooses its optimal signal power that maximizes its payoff. 
In addition, the energy of these wireless radios is assumed to be limited. Therefore, players will not choose an over sized power to emit a signal, because of the impact of the increasing transmission cost on their payoffs.

\section{AMJPC EQUILIBRIUMS}

Move on now to derive the $\mathrm{NE}$ and the $\mathrm{SE}$ in the AMJPC Game. For simplicity, and without loss of generality, we assume the existence of two jammers. Consequently, the utility functions in the Game $G_{3}=$ $\left(\left\{\mathcal{T}, \mathcal{J}_{1}, \mathcal{J}_{2}\right\},\left\{P, J_{1}, J_{2}\right\},\{U, V 1, V 2\}\right)$ are given by the following formulations:

$$
\begin{gathered}
U\left(P, J_{1}, J_{2}\right)=\frac{\alpha P}{N+\sum_{i=1}^{2} \beta_{i} J_{i}}-C P \\
V_{1}\left(P, J_{1}, J_{2}\right)=-\frac{\alpha P}{N+\sum_{i=1}^{2} \beta_{i} J_{i}}-E_{1} J_{1} \\
V_{2}\left(P, J_{1}, J_{2}\right)=-\frac{\alpha P}{N+\sum_{i=1}^{2} \beta_{i} J_{i}}-E_{2} J_{2}
\end{gathered}
$$

\section{A. Nash Game}

Let's assume the presence of two regular jammers which are not eligible to sense the ongoing signal power.

By definition, the $\mathrm{NE}$ is a point where no player can increase its utility function by unilaterally changing its strategy, thus, this Equilibrium denoted by $\left(P^{N E}, J_{1}^{N E}, J_{2}^{N E}\right)$ corresponds to a desirable strategy of the players, namely:

$$
\begin{aligned}
& P^{N E}=\underset{P \geq 0}{\operatorname{argmax}} U\left(P, J_{1}^{N E}, J_{2}^{N E}\right) \\
& J_{1}^{N E}=\underset{J_{1} \geq 0}{\operatorname{argmax}} V 1\left(P^{N E}, J_{1}, J_{2}^{N E}\right) \\
& J_{2}^{N E}=\underset{J_{2} \geq 0}{\operatorname{argmax}} V 2\left(P^{N E}, J_{1}^{N E}, J_{2}\right)
\end{aligned}
$$

Proposition 1: The unique NE strategy of the AMJPC Game, denoted by $\left(P^{N E}, J_{1}^{N E}, J_{2}^{N E}\right)$, respects the following formulations:

$$
\begin{gathered}
P^{N E}= \begin{cases}0 & Q_{1} \\
\frac{\alpha}{C^{2}} \min \left(\frac{1}{\mathcal{J E} \mathcal{R}_{2}}, \frac{1}{\mathcal{J} \mathcal{E} \mathcal{R}_{1}}\right) & \text { ow }\end{cases} \\
\left(J_{1}^{N E}, J_{2}^{N E}\right)= \begin{cases}(0,0) & Q_{1} \\
\left(\frac{\alpha / C-N}{\beta_{1}}, 0\right) & Q_{2} \\
\left(0, \frac{\alpha / C-N}{\beta_{2}}\right) & Q_{3} \\
\left(J^{\prime}, \frac{1}{\beta_{2}}\left(\alpha / C-N-\beta_{1} J^{\prime}\right)\right), & \text { ow } \\
\text { where }, 0 \leq J^{\prime} \leq \frac{1}{\beta_{1}}(\alpha / C-N)\end{cases}
\end{gathered}
$$

whereas the corresponding utility values are:

$$
U^{N E}=0
$$

$$
\begin{aligned}
& \left(V_{1}^{N E}, V_{2}^{N E}\right)= \\
& \begin{cases}(0,0) & Q_{1} \\
\left(\frac{1}{\mathcal{J E R}_{1}}(N-2 \alpha / C),-\frac{1}{\mathcal{J E R} \mathcal{R}_{1}} \frac{\alpha}{C}\right. & Q_{2} \\
\left(-\frac{1}{\mathcal{J} \mathcal{E} \mathcal{R}_{2}} \frac{\alpha}{C}, \frac{1}{\mathcal{J E} \mathcal{R}_{2}}(N-2 \alpha / C)\right. & Q_{3} \\
\left(\frac{1}{\mathcal{J E R}_{1}}\left(-\alpha / C-\beta_{1} J^{\prime}\right),\right. & \\
\left.\frac{1}{\mathcal{J} \mathcal{R}_{2}}\left(N-2 \alpha / C+\beta_{1} J^{\prime}\right)\right), & \\
\text { where }, 0 \leq J^{\prime} \leq \frac{1}{\beta_{1}}(\alpha / C-N) & \text { ow }\end{cases}
\end{aligned}
$$

the conditions are given by:

- $Q_{1}: \frac{\alpha}{C} \leq N$

- $\quad Q_{2}: \frac{\alpha}{C}>N, \mathcal{J} \mathcal{E} \mathcal{R}_{2}<\mathcal{J} \mathcal{E} \mathcal{R}_{1}$

- $\quad Q_{3}: \frac{\alpha}{C}>N, \mathcal{J} \mathcal{E} \mathcal{R}_{2}>\mathcal{J} \mathcal{E R}_{1}$

It turns out from Proposition 1 that, when the condition $Q_{1}$ is not satisfied (Eq. (9)), the attack is lunched by the jammer that has the highest $\mathcal{J E} \mathcal{R}$ value, while the other one is inactive. Furthermore, if the two jammers share the same $\mathcal{J E R}$, then the cumulative attack is initiating by the two jammers so as to carry out a single attack seeming to come from the jammer that has the highest $\mathcal{J E R}$ value (i.e., both jammers cooperate with each other).

\section{B. Stackelberg Game}

Let's consider two smart jammers that have the intelligence to rapidly learn the transmitter signal power and adjust accordingly their owns. Based on the fact that the jammers take action if and only if the channel is sensed to be busy, SE is the appropriate strategy against these smart jamming behaviors. Thus, this subsection focuses in deriving the AMJPC SE in which the transmitter is the leader and the jammers represent the set of followers. In this Stackleberg Game, the follower's Game is played after the leader Game, and its outcome depends on the action of the leader. The leader fixes its optimal strategy based on the reaction of the followers and lets them optimize their own utility according to the leader strategy.

1) Jammers's Optimal Strategy: Taking into account the transmitter's strategy, the jammers's optimal strategy is computed by solving the following maximization problem:

$$
\begin{aligned}
& \max _{J_{1} \geq 0} V_{1}\left(P, J_{1}, J_{2}\right) ; \forall P \geq 0, \forall J_{2} \geq 0 \\
& \max _{J_{2} \geq 0} V_{2}\left(P, J_{1}, J_{2}\right) ; \forall P \geq 0, \forall J_{1} \geq 0
\end{aligned}
$$

Proposition 2: Let $\mathrm{P}$ be the ongoing signal power of the transmitter, then, the corresponding optimal strategy $\hat{J}=$ $\left(\hat{J}_{1}, \hat{J}_{2}\right)$ of the two jammers respects the following formulation:

$$
\begin{aligned}
& \left(\hat{J}_{1}, \hat{J}_{2}\right)(P)= \\
& \begin{cases}(0,0) & C_{1} \\
\left(\frac{1}{\beta_{1}}\left(\sqrt{\alpha P \cdot \mathcal{J E} \mathcal{R}_{1}}-N\right), 0\right) & C_{2} \\
\left(0, \frac{1}{\beta_{2}}\left(\sqrt{\alpha P \cdot \mathcal{J E} \mathcal{R}_{2}}-N\right)\right) & C_{3} \\
\left(J^{\prime}, \frac{1}{\beta_{2}}\left(\sqrt{\alpha P \cdot \mathcal{J E} \mathcal{R}_{2}}-N-\beta_{1} J^{\prime}\right)\right), & \\
\text { where }, 0 \leq J^{\prime} \leq \frac{1}{\beta_{1}}\left(\sqrt{\alpha P \cdot \mathcal{J} \mathcal{E} \mathcal{R}_{1}}-N\right) & \text { ow }\end{cases}
\end{aligned}
$$

whereas the corresponding utility value $V(P)=\left(V_{1}, V_{2}\right)(P)$ of the two jammers is: 


$$
\begin{aligned}
& V(P)= \\
& \begin{cases}\left(-\frac{\alpha P}{N},-\frac{\alpha P}{N}\right) & C_{1} \\
\left(\left(N-2 \sqrt{\alpha P \cdot \mathcal{J E} \mathcal{R}_{1}}\right) / \mathcal{J E} \mathcal{R}_{1},-\sqrt{\frac{\alpha P}{\mathcal{J E R}}}\right) & C_{2} \\
\left(-\sqrt{\frac{\alpha P}{\mathcal{J E R} \mathcal{R}_{2}}},\left(N-2 \sqrt{\alpha P . \mathcal{J E} \mathcal{R}_{2}}\right) / \mathcal{J E} \mathcal{R}_{2}\right) & C_{3} \\
\left(-\left(\sqrt{\alpha P \cdot \mathcal{J} \mathcal{E} \mathcal{R}_{1}}+\beta_{1} J^{\prime}\right) / \mathcal{J E} \mathcal{R}_{1},\right. & \\
\left.\left(-2 \sqrt{\alpha P . \mathcal{J E} \mathcal{R}_{2}}+N+\beta_{1} J^{\prime}\right) / \mathcal{J} \mathcal{E} \mathcal{R}_{2}\right), & \\
\text { where }_{0} \leq J^{\prime} \leq \frac{1}{\beta_{1}}\left(\sqrt{\alpha P . \mathcal{J} \mathcal{E} \mathcal{R}_{1}}-N\right) & \text { ow }\end{cases}
\end{aligned}
$$

the conditions are given by:

- $\quad C_{1}: \frac{\alpha P}{N^{2}} \leq \min \left(\frac{1}{\mathcal{J} \mathcal{E} \mathcal{R}_{1}}, \frac{1}{\mathcal{J E} \mathcal{R}_{2}}\right)$

- $\quad C_{2}: \frac{\alpha P}{N^{2}}>\frac{1}{\mathcal{J E} \mathcal{R}_{1}}, \mathcal{J E} \mathcal{R}_{1}>\mathcal{J} \mathcal{E} \mathcal{R}_{2}$

- $\quad C_{3}: \frac{\alpha P}{N^{2}}>\frac{1}{\mathcal{J} \mathcal{E} \mathcal{R}_{2}}, \mathcal{J} \mathcal{E} \mathcal{R}_{1}<\mathcal{J} \mathcal{E} \mathcal{R}_{2}$

2) Transmitter's Optimal Strategy: The transmitter can predict the jammer's reaction based on Proposition 2, therefore, the optimal transmitter's strategy is computed by solving the following maximization problem:

$$
\max _{P \geq 0} U\left(P, \hat{J}_{1}(P), \hat{J}_{2}(P)\right)
$$

Proposition 3: The optimal strategy of the transmitter is:

$$
P^{S E}= \begin{cases}0 & R_{1} \\ \frac{N^{2}}{\alpha} \cdot \max \left(\mathcal{J E} \mathcal{R}_{1}, \mathcal{J E} \mathcal{R}_{2}\right) & R_{2} \\ \frac{\alpha}{4 C^{2}} \cdot \max \left(\mathcal{J E} \mathcal{R}_{1}, \mathcal{J E} \mathcal{R}_{2}\right) & \text { ow }\end{cases}
$$

whereas the corresponding utility value $U^{S E}=$ $U\left(P^{S E}, J_{1}\left(P^{S E}\right), J_{2}\left(P^{S E}\right)\right)$ is:

$$
U^{S E}= \begin{cases}0 & R_{1} \\ (\alpha-C N) \frac{N}{\alpha} \cdot \max \left(\mathcal{J} \mathcal{E} \mathcal{R}_{1}, \mathcal{J} \mathcal{E} \mathcal{R}_{2}\right) & R_{2} \\ \frac{\alpha}{4 C} \cdot \max \left(\mathcal{J E} \mathcal{R}_{1}, \mathcal{J} \mathcal{E} \mathcal{R}_{2}\right) & \text { ow }\end{cases}
$$

the conditions are given by:

- $R_{1}: \frac{\alpha}{C} \leq N$

- $R_{2}: N<\frac{\alpha}{C} \leq 2 N$

In conclusion, according to Eq. (18), the transmitter as a leader selects its signal power in overall consideration of the impact on both jammers' reaction. If the transmission cost of the transmitter is sufficiently high (i.e., the R1 condition is satisfied), the transmitter's optimal anti-reaction is to stop the transmission activity; otherwise, the optimal one is when the transmitter adjusts its strategy based on all channel gains, channel noise, transmission cost and the jamming cost of both jammers.

Corollary 1: The 3-tuple $\left(P^{S E}, \hat{J}_{1}\left(P^{S E}\right), \hat{J}_{2}\left(P^{S E}\right)\right)$ is the SE of the AMJPC Game, where :

$$
P^{S E}= \begin{cases}0 & R_{1} \\ \frac{N^{2}}{\alpha} \cdot \max \left(\mathcal{J} \mathcal{E} \mathcal{R}_{1}, \mathcal{J} \mathcal{E} \mathcal{R}_{2}\right) & R_{2} \\ \frac{\alpha}{4 C^{2}} \cdot \max \left(\mathcal{J E} \mathcal{R}_{1}, \mathcal{J E} \mathcal{R}_{2}\right) & \text { ow }\end{cases}
$$

$$
\begin{gathered}
\left(J_{1}^{S E}, J_{2}^{S E}\right)= \\
\begin{cases}(0,0) & R_{1}, R_{2} \\
\left(\frac{1}{\beta_{1}}\left(\frac{\alpha}{2 C}-N\right), 0\right) & R_{3}, \mathcal{J} \mathcal{E} \mathcal{R}_{1}>\mathcal{J E} \mathcal{R}_{2} \\
\left(0, \frac{1}{\beta_{2}}\left(\frac{\alpha}{2 C}-N\right)\right) & R_{3}, \mathcal{J} \mathcal{E} \mathcal{R}_{1}<\mathcal{J E} \mathcal{R}_{2} \\
\left(J^{\prime}, \frac{1}{\beta_{2}}\left(\frac{\alpha}{2 C}-N-\beta_{1} J^{\prime}\right)\right), & \\
\text { where }, 0 \leq J^{\prime} \leq \frac{1}{\beta_{1}}\left(\frac{\alpha}{2 C}-N\right) & \text { ow }\end{cases}
\end{gathered}
$$

whereas the corresponding utility values

$$
\begin{aligned}
& U^{S E}= \begin{cases}0 & R_{1} \\
(\alpha-C N) \frac{N}{\alpha} \cdot \max \left(\mathcal{J} \mathcal{E} \mathcal{R}_{1}, \mathcal{J} \mathcal{E} \mathcal{R}_{2}\right) & R_{2} \\
\frac{\alpha}{4 C} \cdot \max \left(\mathcal{J} \mathcal{E} \mathcal{R}_{1}, \mathcal{J} \mathcal{E} \mathcal{R}_{2}\right) & \text { ow }\end{cases} \\
& \left(V_{1}^{S E}, V_{2}^{S E}\right)= \\
& \begin{cases}(0,0) & R_{1} \\
\left(-N \cdot \max \left(\mathcal{J} \mathcal{E} \mathcal{R}_{1}, \mathcal{J} \mathcal{E} \mathcal{R}_{2}\right),\right. & \\
\left.-N \cdot \max \left(\mathcal{J} \mathcal{E} \mathcal{R}_{1}, \mathcal{J} \mathcal{E} \mathcal{R}_{2}\right)\right) & R_{2} \\
\left(\left(N-\frac{\alpha}{C}\right) / \mathcal{J E} \mathcal{R}_{1},-\frac{\alpha}{2 C . \mathcal{J E R}_{1}}\right) & R_{3}, \mathcal{J} \mathcal{E} \mathcal{R}_{1}>\mathcal{J} \mathcal{E} \mathcal{R}_{2} \\
\left(-\frac{\alpha}{2 C . \mathcal{J} \mathcal{E} \mathcal{R}_{2}},\left(N-\frac{\alpha}{C}\right) / \mathcal{J E}_{2}\right) & R_{3}, \mathcal{J} \mathcal{E} \mathcal{R}_{1}<\mathcal{J} \mathcal{E} \mathcal{R}_{2} \\
\left(-\frac{\alpha}{2 C . \mathcal{J} \mathcal{E} \mathcal{R}_{1}}-\beta_{1} J^{\prime} / \mathcal{J} \mathcal{E} \mathcal{R}_{1},\right. & \\
\left.\left(N+\beta_{1} J^{\prime}-\frac{\alpha}{C}\right) / \mathcal{J} \mathcal{E} \mathcal{R}_{2}\right) & \\
\text { where }, 0 \leq J^{\prime} \leq \frac{1}{\beta_{1}}\left(\frac{\alpha}{2 C}-N\right) & \text { ow }\end{cases}
\end{aligned}
$$

the conditions are given by:

- $R_{1}: \frac{\alpha}{C} \leq N$

- $R_{2}: N<\frac{\alpha}{C} \leq 2 N$

- $R_{3}: \frac{\alpha}{C}>2 N$

The above Corollary proves that, on the transmitter side, the most threatening jammer is the one which has the highest $\mathcal{J E R}$. This result is due to the fact that this particular jammer plays the role of an active player in the Game, whereas, the other one remains in standby mode.

Corollary 2: Let the $S I N R^{S E}$ and $S I N R^{N E}$ be the SINR of the transmitter at $\mathrm{SE}$ and NE respectively. Let $P^{S E}$ and $P^{N E}$ be the transmitter signal power at $\mathrm{SE}$ and $\mathrm{NE}$ respectively. For all $\alpha, C, \beta_{i}, E_{i}$ and $N$ we have the following mathematical inequality:

$$
\left\{\begin{array}{l}
U^{S E} \geq U^{N E} \\
V_{i}^{S E} \geq V_{i}^{N E} \\
S I N R^{S E} \leq S I N R^{N E} \\
P^{S E} \leq P^{N E}
\end{array} \quad i \in\{1,2\}\right.
$$

Based on the Corollary 2, it's clear that, the transmitter gains in terms of power in the presence of smart jammers, whereas, it gains in terms of SINR in the presence of regular jammers.

\section{Simulation Results}

\section{A. AMJPC Scheme's Performance}

Let's move on now to evaluate jamming-resistance against Multiple Jamming attacks (MJs). Note that the case of Single Jamming attack (SJ) in [17],[18] and [19] can be deduced from the proposed AMJPC Game model. The system variables used 


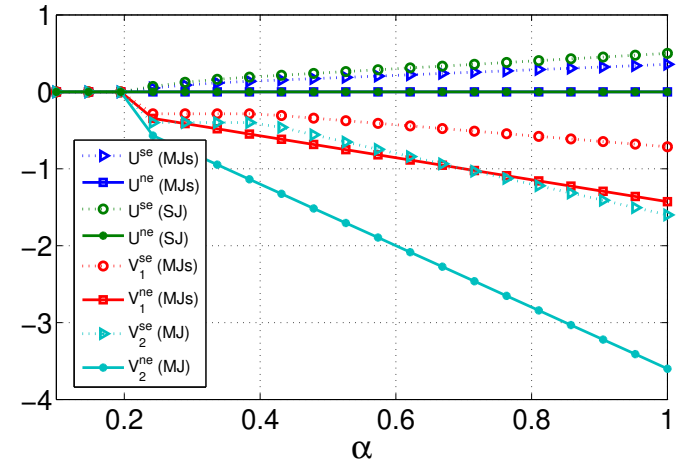

Fig. 2. Utility Functions in both SE and NE with respect to $\alpha$ in the two cases: SJ and MJs.

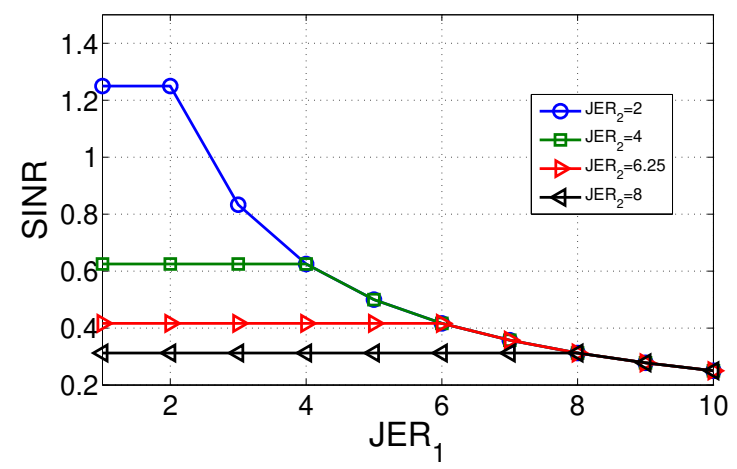

Fig. 3. Transmitter's SINR in SE with respect to $\mathcal{J E} \mathcal{R}_{1}$ for different $\mathcal{J E} \mathcal{R}_{2}$ values.

to depict the numerical results are given by : $C=E_{1}=E_{2}=$ $0.1, N=2$ and $\left(\alpha, \beta_{1}, \beta_{2}\right) \in[0.1,1]^{3}$.

Fig. 2 describes the impact of parameter $\alpha$ on the players' utilities for the following scenarios: 1)-The presence of SJ with $\beta_{1}=0.5$. 2)-The presence of MJs with $\beta_{1}=0.5$ and $\beta_{2}=0.7$. In this figure, SE leads to higher utilities than NE does. Hence, as $\alpha$ increases, the transmitter's SE payoff is more improved while the jammers' SE payoff decreases. The intuitive reason is that the larger $\alpha$ became, the better the transmitter channel gain is. In addition, the jammers' utility in the SE strategy is higher than the one in NE strategy, because, in the SE strategy, the smart jammers can quickly learn the legitimate signal power before making a decision.

Many other observations can be made, for example, when the fading channel gain of the transmitter is $\alpha=1$, its utility in the presence of a SJ is 0.5 in SE strategy and 0 in NE strategy, while in the presence of MJs, it is only 0.3571 in SE strategy and 0 in NE strategy; (note that 0.3571 corresponds to a SE utility in presence of SJ with $\beta_{1}=0.7$ ). In addition, on the jammers side, SE and NE utility in the MJs scheme are, respectively, higher than SE and NE utility in the SJ scheme. Furthermore, since $\mathcal{J E R}_{2}>\mathcal{J E R}_{1}, \mathcal{J}_{2}$ acts as an active jammer in the Game, whereas, the other one is inactive. This behavior enhances the jamming performance especially whenever jammers have a high cost or located far from the receiver. Thus, the transmitter will consider only the presence of the jammer that has the highest Jamming Efficiency Ratio.
In order to have a closer look on the impact of $\mathcal{J E R}$ on the SINR of the transmitter, Fig. 3 depicts the transmitter's SINR in the SE with respect to $\mathcal{J E} \mathcal{R}_{1}$ in the MJs scheme for different $\mathcal{J} \mathcal{E} \mathcal{R}_{2}$ values. It's easy to remark that, from the transmitter viewpoint, the most dangerous jammer is the one which has the highest $\mathcal{J E R}$.

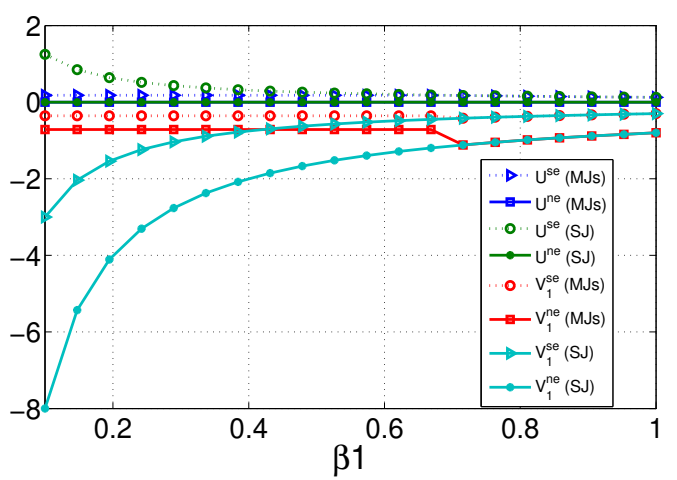

Fig. 4. Utility Functions in both SE and NE with respect to $\beta$ in the two cases: SJ and MJs.

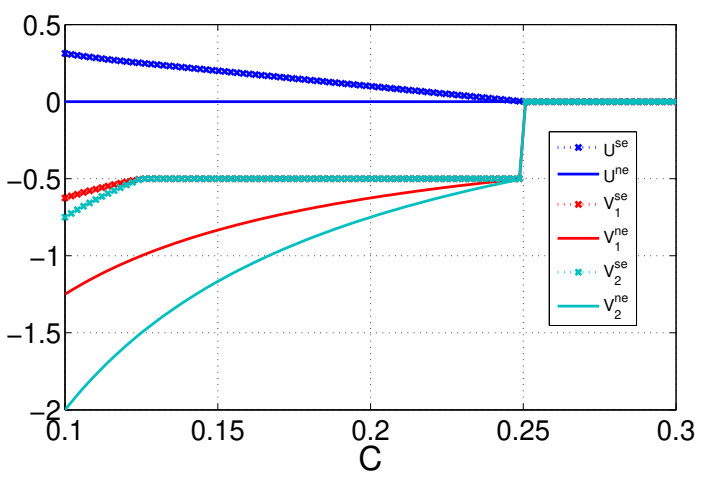

Fig. 5. Utility function in both SE and NE with respect to $C$ for $\beta_{1}=0.3, \beta_{2}=$ $0.6, \alpha=0.5$

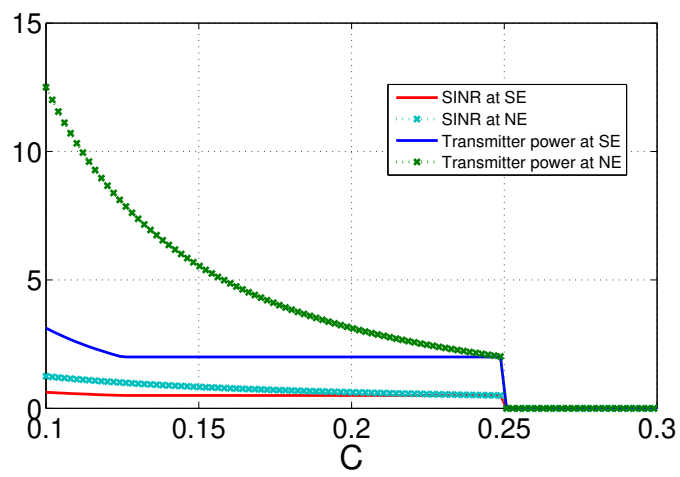

Fig. 6. SINR and transmission power of the transmitter in both SE and NE with respect to $C$ for $\beta_{1}=0.3, \beta_{2}=0.6, \alpha=0.5$.

Let's move on now to investigate the impact of $\beta_{1}$ on the players' utilities in the two following cases: SJ with $\beta_{1}=0.5$, and MJs with $\beta_{1}=0.5$ and $\beta_{2}=0.7$. As can be noticed from Fig. 4, the SE leads to higher utilities for all players more than NE does. In addition, as $\beta_{1}$ increases, the transmitter's 
SE payoff decreases while the jammers' SE payoff increases, this is due to the fact that the larger $\beta_{1}$ became, the better the channel gain of $\mathcal{J}_{1}$ is. On the other hand, this figure can be split into two parts. Let's denote the first part by Part.1 when $\beta_{1}<0.7$ and the second by Part.2 when $\beta_{1} \geq 0.7$. In the Part.1, the NE and SE utilities of players for MJs are fixed to 0.1786 for $U^{S E}(M J s), 0.3571$ for $V_{1}^{S E}(M J s), 0$ for $U^{N E}(M J s)$ and 0.7143 for $V_{1}^{N E}(M J s)$. The NE and SE utilities of the transmitter in the SJ case are higher than in the MJs case; also, the NE and SE utilities of $\mathcal{J}_{1}$ in the SJ case are lower than in the MJs case. This is due to the fact that, as $\mathcal{J E R}_{2}<\mathcal{J} \mathcal{E} \mathcal{R}_{1}, \mathcal{J}_{1}$ behaves like an inactive one. As for, contrary to Part.1, Part.2 shows that $\mathcal{J}_{1}$ Influences the utility of all players and acts as an active one. Note that in Part.2, the utilities of all players in the MJs scheme coincide with the utilities in SJ scheme.

Fig. 5 describes the impact of the transmitter' transmission cost on the players' utilities in NE and SE, with $\beta_{1}=0.3, \beta_{2}=$ 0.6 and $\alpha=0.5$. Hence, as $C$ increases, the transmitter's SE/NE utilities decrease while the jammers' SE/NE utilities increase. This phenomenon is due to the fact that the larger $C$ became, the more the transmitter has no interest in transmitting the signal so as to conserve its battery life. Thereafter, the larger $C$ became, the more jammers have no interest in jamming the communication. In addition, from a certain value of $C\left(\frac{\alpha}{N}=0.25\right)$, all players (transmitter and jammers) go into standby mode with $U^{S E}=U^{N E}=0, V_{i}^{S E}=V_{i}{ }^{N E}=0$, $\forall i \in\{1,2\}$.

Fig. 6 describes the impact of the transmitter' transmission cost on the transmitter' SINR and transmission power in NE and SE, with $\beta_{1}=0.3, \beta_{2}=0.6$ and $\alpha=0.5$. Hence, as $C$ increases, the $S I N R^{S E}, S I N R^{N E}, P^{S E}$ and $P^{N E}$ decrease in order to economize the available transmitter power. Thereafter, from a certain value of $C\left(\frac{\alpha}{N}=0.25\right)$, the transmitter becomes inactive $\left(P^{S E}=P^{N E}=0\right)$. In addition, NE scheme leads to higher SINR and transmission power than SE scheme does. This is due to the fact that, in the SE strategy, the transmitter adjusts its transmission power according to the reaction prediction of the jammers which can quickly learn the legitimate signal power before making a decision. Moreover, the communication is seriously more destroyed in SE strategy than in NE strategy; Thus, the transmitter gains in terms of power in SE scheme, whereas, it gains in terms of SINR in the NE scheme.

\section{B. AMJPC Model with an Incomplete Information}

In order to have a closer look on the impact of an incomplete knowledge about the dynamic environment, let's consider a scenario where the AMJPC strategy is selected based on the Q-learning technique developed in [20].

Fig. 8 depicts the transmitter payoff received by the receiver, and Fig. 7 depicts the jammers payoffs, where the transmitter selects its signal power based on the Q-learning method. From the two figures, it's clear that all players payoff converges towards the solution proved in the closed form expressions of the above model. This validates the proposed AMJPC scheme. In addition, Fig. 8 proves that the transmitter is gradually aware of the dynamic environment with the learning episodes increasing, which indicates a well jamming-resistance. This is due to the fact that the transmitter chooses a more optimal signal power action after having a well knowledge about the environment.

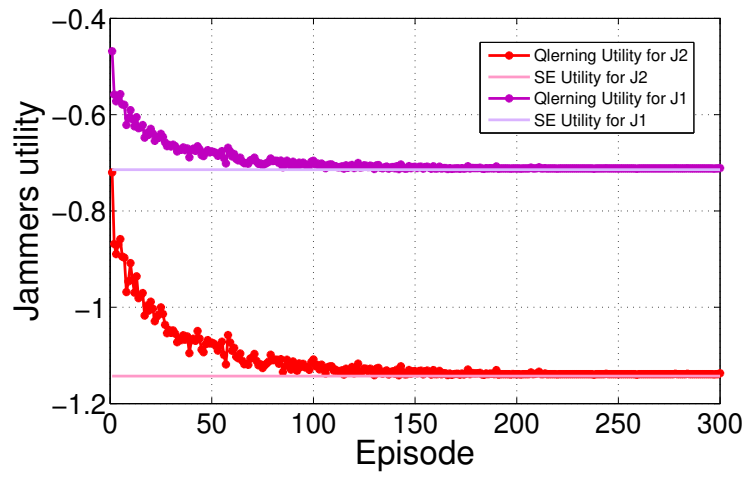

Fig. 7. Jammers utility where the transmitter chooses its transmission power based on Q-learning.

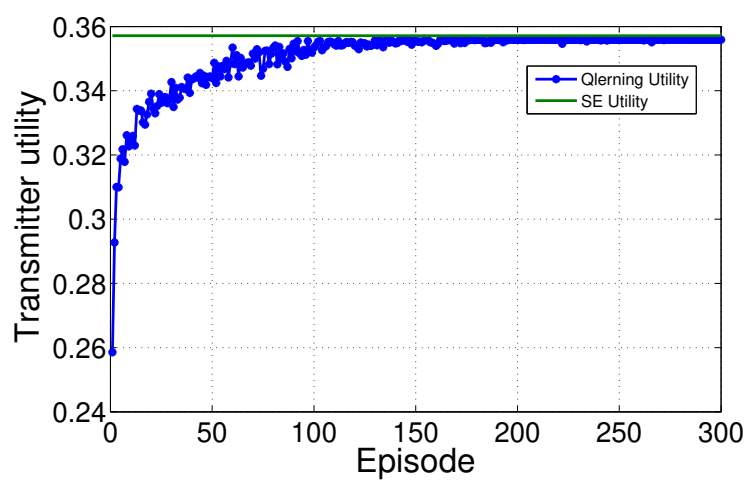

Fig. 8. Transmitter utility where the transmission power is chosen based on Q-learning.

\section{CONCLUSION}

This paper proposed an Anti Multi-Jamming based Power Control in the presence of several smart and regular jammers from a Game theoretical point of view. It proved the existence and uniqueness of NE and SE and provided analytic expressions for the equilibrium strategies. Moreover. It turned out that the jammer which has the highest Jamming Efficiency Ratio plays the role of an active player in the Game, whereas, the other one becomes standby. Thus, from the transmitter viewpoint, the AMJPC Game is reduced to an anti-jamming Game under a single jammer which has the highest $\mathcal{J E R}$, this jammer is considered as the only hazardous jammer for the transmitter. Finally, by means of simulation results, the transmitter can efficiently improve the jamming-resistance. Furthermore, the transmitter gains in terms of power in the presence of smart jammers, whereas, it gains in terms of SINR in the presence of regular jammers. As a future scope, the considered battle "one transmitter- several jammers" can be extended to the battle "one transmitter-several aggressive transmitters-several jammers".

\section{APPENDIX}

Proof of Proposition 1: Let $i \in[1,2]$. 
The first order partial derivative of the jammer $i$ objective function with respect to $J_{i}$ is:

$$
\frac{\partial V_{i}}{\partial J_{i}}=\frac{\alpha \beta_{i} P}{\left(N+\beta_{1} J_{1}+\beta_{2} J_{2}\right)^{2}}-E_{i}
$$

The second order partial derivatives of the jammer $i$ objective function is:

$$
\frac{\partial^{2} V_{i}}{\partial J_{i}^{2}}=\frac{-\alpha \beta_{i}^{2} P}{\left(N+\beta_{1} J_{1}+\beta_{2} J_{2}\right)^{3}} ;
$$

According to Eq. (26), $V_{i}$ is strictly concave in $J_{i}$.

Assume that the transmitter has fixed its strategy, so the transmitter is now an inactive player and it wishes knowing which utility it can get under the most unfavorable circumstances. Therefore, by setting the Eq. (25) to 0 based on the fact that $J_{i} \geq 0$, the jammer's optimal strategies $\hat{J}_{1}$ and $\hat{J}_{2}$ respect the following two equations :

$$
\begin{aligned}
& \hat{J}_{1}=\max \left(0, \frac{1}{\beta_{1}}\left(\sqrt{\frac{\alpha \beta_{1} P}{E_{1}}}-\left(N+\beta_{2} J_{2}\right)\right)\right), \forall J_{2} \geq 0 . \\
& \hat{J}_{2}=\max \left(0, \frac{1}{\beta_{2}}\left(\sqrt{\frac{\alpha \beta_{2} P}{E_{2}}}-\left(N+\beta_{1} J_{1}\right)\right)\right), \forall J_{1} \geq 0 .
\end{aligned}
$$

Assume that $\hat{J}_{1} \hat{J}_{2} \neq 0$. From Eq. (27) and Eq. (28), so: $N+\beta_{1} \hat{J}_{1}+\beta_{2} \hat{J}_{2}=\sqrt{\frac{\alpha \beta_{1} P}{E_{1}}}=\sqrt{\frac{\alpha \beta_{2} P}{E_{2}}}$, yielding $\frac{E_{1}}{\beta_{1}}=\frac{E_{2}}{\beta_{2}}$. Thus:

$$
\frac{E_{1}}{\beta_{1}} \neq \frac{E_{2}}{\beta_{2}} \Longrightarrow \hat{J}_{1} \hat{J}_{2}=0
$$

To compute the NE let's consider the following disjoint cases:

- $Q_{1}: \frac{\alpha}{C} \leq N$ :

The derivative of Eq. (5) with respect to $P$ is $\frac{\partial U}{\partial P}=$ $\frac{\alpha}{\left(N+\beta_{1} J_{1}+\beta_{2} J_{2}\right)}-C \leq 0$.Thus $P^{N E}=0$. on the other hand, $\frac{\partial V_{1}}{\partial J_{1}}\left(0, J_{1}, J_{2}\right)=-E_{1}<0$ and $\frac{\partial V_{2}}{\partial J_{2}}\left(0, J_{1}, J_{2}\right)=-E_{2}<0$, then, $J_{1}^{N E}=J_{2}^{N E}=0$.

- $Q_{2}: \frac{\alpha}{C}>N$ :

○ $\frac{E_{1}}{\beta_{1}}<\frac{E_{2}}{\beta_{2}}$ :

Let $J_{1}^{N E}=\frac{\alpha / C-N}{\beta_{1}}$, as $J_{2}^{N E}=0$ from Eq. (29), then, $\forall P \geq 0, U\left(P, J_{1}^{N E}, J_{2}^{N E}\right)=0$. In order to have $\overline{J_{1}^{N} E}=\frac{\alpha / C-N}{\beta_{1}}$, we must have $P=\frac{\alpha E_{1}}{C^{2} \beta_{1}}$ according to Eq. (27).

Thus, $J_{2}^{N E}=0, J_{1}^{N E}=\frac{1}{\beta_{1}}(\alpha / C-N)$ and $P^{N E}=\frac{\alpha E_{1}}{C^{2} \beta_{1}}$.

Let now prove the uniqueness of this NE for all three players. First, let's assume that there exist an other NE $\left(T^{\prime}, J_{1}^{\prime}, J_{2}^{\prime}\right)$ and let's prove that $T^{\prime}=T^{N E}, J_{1}^{\prime}=J_{1}^{N E}$ and $J_{2}^{\prime}=J_{2}^{N E}$.

- Let $J_{2}^{\prime}>0$, thus from Eq. (28) and (27) we deduce that $J_{1}^{\prime}=\frac{1}{2 \beta_{1}}\left(\sqrt{\frac{\alpha \beta_{1} P^{\prime}}{E_{1}}}-\right.$ $\left.\sqrt{\frac{\alpha \beta_{2} P^{\prime}}{E_{2}}}\right)$, since $\frac{E_{1}}{\beta_{1}}<\frac{E_{2}}{\beta_{2}}$, then $J_{1}^{\prime}>0$ contradicting to Eq. (29). Thus $J_{2}^{\prime}=$ $J_{2}^{N E}=0$.
- $\quad$ Let $J_{1}^{\prime}>J_{1}^{N E}$, then $\frac{\partial U}{\partial P}<0$ yielding $T^{\prime}=0$, then $J_{1}^{\prime}=0$ from Eq. (25), contradicting to the assumption that $J_{1}^{\prime}>J_{1}^{N E}>0$. Thus $J_{1}^{\prime} \leq J_{1}^{N E}$.

* Let $J_{1}^{\prime}<J_{1}^{N E}$, then $\frac{\partial U}{\partial P}>0$ yielding that the transmitter can increase its utility by unilateral deviation, contradicting to the NE concept. Thus $J_{1}^{\prime}=J_{1}^{N E}$.

- From Eq. (27): $T^{\prime}=T^{N E}$.

○ $\frac{E_{1}}{\beta_{1}}>\frac{E_{2}}{\beta_{2}}$ :

By symmetrical approach we deduce that $P^{N E}=\frac{\alpha E_{2}}{C^{2} \beta_{2}}, J_{1}^{N E}=0, J_{2}^{N E}=\frac{1}{\beta_{2}}(\alpha / C-$ $N)$.

○ $\frac{E_{1}}{\beta_{1}}=\frac{E_{2}}{\beta_{2}}$ :

Let $\beta_{1} J_{1}^{N E}+\beta_{2} J_{2}^{N E}=\alpha / C-N$, then, $\forall P \geq 0, U\left(P, J_{1}^{N E}, J_{2}^{N E}\right)=0$. In order to have $\beta_{1} J_{1}^{N E}+\beta_{2} J_{2}^{N E^{2}}=\alpha / C-N$, we must have $P=\frac{\alpha E_{1}}{C^{2} \beta_{1}}$ according to Eq. $(27,28)$.

Thus, $P^{N E}=\frac{\alpha E_{1}}{C^{2} \beta_{1}}$ and $\beta_{1} J_{1}^{N E}+\beta_{2} J_{2}^{N E}=$ $\alpha / C-N$, with $0 \leq J_{i}^{N E} \leq \frac{1}{\beta_{i}}(\alpha / C-N)$.

Move on now to prove the uniqueness of the NE. First, Let's assume that there exist an other NE $\left(T^{\prime}, J_{1}^{\prime}, J_{2}^{\prime}\right)$ and Let's prove that $T^{\prime}=T^{N E}, \beta_{1} J_{1}^{\prime}+\beta_{2} J_{2}^{\prime}=\beta_{1} J_{1}^{N E}+\beta_{2} J_{2}^{N E}$.

- Let $\beta_{1} J_{1}^{\prime}+\beta_{2} J_{2}^{\prime}>\beta_{1} J_{1}^{N E}+\beta_{2} J_{2}^{N E}$, then $\frac{\partial U}{\partial P}<0$ yielding $T^{\prime}=0$, then from Eq. (25) $J_{1}^{\prime}=J_{2}^{\prime}=0$, contradicting to the assumption that $\beta_{1} J_{1}^{\prime}+$ $\beta_{2} J_{2}^{\prime}>\beta_{1} J_{1}^{N E}+\beta_{2} J_{2}^{N E}>0$. Thus $\beta_{1} J_{1}^{\prime}+\beta_{2} J_{2}^{\prime} \leq \beta_{1} J_{1}^{N E}+\beta_{2} J_{2}^{N E}$.

- Let $\beta_{1} J_{1}^{\prime}+\beta_{2} J_{2}^{\prime}<\beta_{1} J_{1}^{N E}+\beta_{2} J_{2}^{N E}$, then $\frac{\partial U}{\partial P}>0$ yielding that the transmitter can increase its utility by unilateral deviation, contradicting to the NE concept. Thus $\beta_{1} J_{1}^{\prime}+\beta_{2} J_{2}^{\prime}=\beta_{1} J_{1}^{N E}+\beta_{2} J_{2}^{N E}$.

- From Eq. (27), $T^{\prime}=T^{N E}$.

Proof of Proposition 2: Consider Eq. (27) and Eq. (28).

In order to compute the optimal jamming power of both jammer $i$ with respect to $P$, let's consider the following disjoint cases:

- $\quad \frac{\alpha P}{N^{2}} \leq \min \left(\frac{E_{1}}{\beta_{1}}, \frac{E_{2}}{\beta_{2}}\right)$ :

From Eq. $(27,28), \hat{J}_{1}=\hat{J}_{2}=0$.

- $\quad \frac{\alpha P}{N^{2}}>\min \left(\frac{E_{1}}{\beta_{1}}, \frac{E_{2}}{\beta_{2}}\right)$ :

○ $\frac{E_{1}}{\beta_{1}}<\frac{E_{2}}{\beta_{2}}$ :

- $\min \left(\frac{E_{1}}{\beta_{1}}, \frac{E_{2}}{\beta_{2}}\right)<\frac{\alpha P}{N^{2}} \leq \operatorname{Max}\left(\frac{E_{1}}{\beta_{1}}, \frac{E_{2}}{\beta_{2}}\right)$ :

From Eq. (28), $\hat{J}_{2}=0$. by plugging the $\hat{J}_{2}$ value into Eq. (27) we deduce $\hat{J}_{1}=$ $\frac{1}{\beta_{1}}\left(\sqrt{\frac{\alpha \beta_{1} P}{E_{1}}}-N\right)$.

- $\quad \frac{\alpha P}{N^{2}}>\max \left(\frac{E_{1}}{\beta_{1}}, \frac{E_{2}}{\beta_{2}}\right)$ :

Let first prove that $\hat{J}_{1} \neq 0$.

Assume to the contrary that $\hat{J}_{1}=0$. By plugging the value of $\hat{J}_{1}$ into Eq. (28), we have $\hat{J}_{2}=\frac{1}{\beta_{2}}\left(\sqrt{\frac{\alpha \beta_{2} P}{E_{2}}}-N\right)$. Since 


$$
\begin{aligned}
& \frac{E_{1}}{\beta_{1}}<\frac{E_{2}}{\beta_{2}} \text {, thus } \frac{\alpha P}{\left(N+\beta_{2} \hat{J}_{2}\right)^{2}}>\frac{E_{1}}{\beta_{1}} \text {, yielding } \\
& \hat{J}_{1}>0 \text {, contradicting to the assumption } \\
& \text { that } \hat{J}_{1}=0 \text {. Thus } \hat{J}_{1} \neq 0 \text {. } \\
& \text { From Eq. (29), } \hat{J}_{2}=0 \text {. } \\
& \text { Thus, } \hat{J}_{2}=0 \text { and } \hat{J}_{1}=\frac{1}{\beta_{1}}\left(\sqrt{\frac{\alpha \beta_{1} P}{E_{1}}}-N\right) \text {. } \\
& \text { - } \quad \frac{E_{1}}{\beta_{1}}>\frac{E_{2}}{\beta_{2}} \text { : } \\
& \text { - } \min \left(\frac{E_{1}}{\beta_{1}}, \frac{E_{2}}{\beta_{2}}\right)<\frac{\alpha P}{N^{2}} \leq \max \left(\frac{E_{1}}{\beta_{1}}, \frac{E_{2}}{\beta_{2}}\right) \text { : }
\end{aligned}
$$

Proof of Proposition 3: Let $G(P)=U\left(P, \hat{J}_{1}(P), \hat{J}_{2}(P)\right)$. By plugging Proposition (2) result into Eq. (5), we have:

$$
\begin{aligned}
& G(P)= \\
& \begin{cases}(\alpha / N-C) P, & \frac{\alpha P}{N^{2}} \leq \max \left(\frac{\beta_{1}}{E_{1}}, \frac{\beta_{2}}{E_{2}}\right), \\
\sqrt{\alpha\left(\max \left(\frac{\beta_{1}}{E_{1}}, \frac{\beta_{2}}{E_{2}}\right)\right) P}-C P, & \text { ow, }\end{cases}
\end{aligned}
$$

From Eq. 30, If $P>P 1=\frac{N^{2}}{\alpha} \cdot \max \left(\frac{\beta_{1}}{E_{1}}, \frac{\beta_{2}}{E_{2}}\right)$, then $\frac{\partial G}{\partial P}=\frac{1}{2} \sqrt{\frac{\alpha}{P} \cdot \max \left(\frac{\beta_{1}}{E_{1}}, \frac{\beta_{2}}{E_{2}}\right)}-C$ and $\frac{\partial^{2} G}{\partial P^{2}}=$

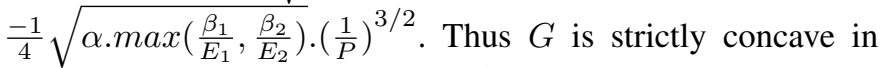
$P$, and $\frac{\partial G}{\partial P}\left(P 0=\frac{\alpha}{4 C^{2}} \cdot \max \left(\frac{\beta_{1}}{E_{1}}, \frac{\beta_{2}}{E_{2}}\right)\right)=0$.

In order to compute the optimal transmitter power given the reaction of the two jammers, let's consider the following three disjoint cases:

- $\quad R_{1}: \frac{\alpha}{C} \leq N$ : In this case, $\frac{P 0}{P 1}=\left(\frac{\alpha}{2 C N}\right)^{2} \leq 1 / 4$, thus, $P 0<P 1$. As shown in Fig. $9\left(R_{3}\right), G(P)$ achieves its maximum when $P=0$.

- $\quad R_{2}: N<\frac{\alpha}{C} \leq 2 N$ : In this case, $\frac{P 0}{P 1}=\left(\frac{\alpha}{2 C N}\right)^{2} \leq 1$ $P 0 \leq P 1$. As shown in Fig. $9\left(R_{2}\right), G(P)$ achieves its maximum when $P=P 1$.

- $\quad R_{3}: \frac{\alpha}{C}>2 N$ : In this case, $P 0>P 1$. As shown in Fig. $9\left(R_{1}\right), G(P)$ achieves its maximum when $P=$ $P 0$.

Proof of Corollary 1: This result can be deduced from Propositions $(2,3)$.

Proof of Corollary 2: From Proposition 1 and Corollary 1, we consider the following disjoint cases :
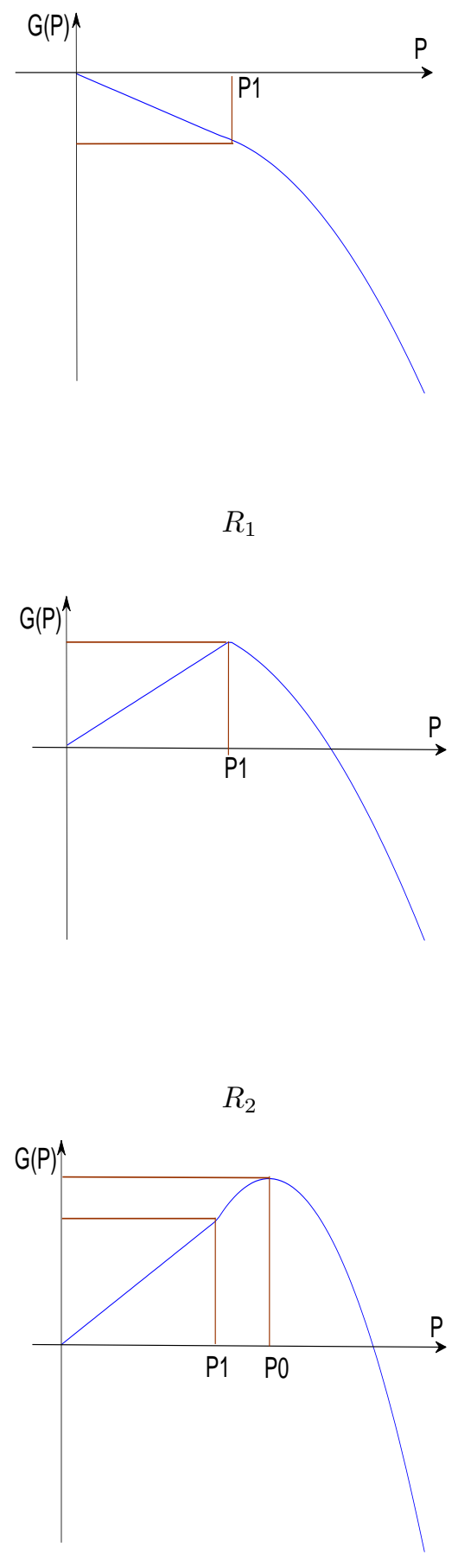

$R_{3}$

Fig. 9. Assumption of $\mathrm{G}(\mathrm{P})$ with respect to $\mathrm{P}$

- $R_{1}: \frac{\alpha}{C}<N$ :

In this case: 
$P^{S E}=P^{N E}, S I N R^{S E}=S I N R^{N E}, U^{S E}=U^{N E}$ $V_{i}{ }^{S E}=V_{i}{ }^{N E}, \forall i \in\{1,2\}$.

- $R_{2}: N \leq \frac{\alpha}{C}<2 N$

$U^{S E}-\bar{U}^{N E}=$

$\left(\frac{\alpha}{C}-N\right) \frac{N C}{\alpha} \cdot \max \left(\mathcal{J E} \mathcal{R}_{1}, \mathcal{J E} \mathcal{R}_{2}\right) \geq 0, P^{S E}-$ $P^{N E}=\frac{1}{\alpha}\left(N^{2}-\left(\frac{\alpha}{C}\right)^{2}\right) \cdot \max \left(\mathcal{J E} \mathcal{R}_{1}, \mathcal{J E} \mathcal{E} \mathcal{R}_{2}\right) \leq 0$ and $S I N R^{S E}-S I N R^{N E}=(N-$ $\left.\frac{\alpha}{C}\right) \cdot \max \left(\mathcal{J E R} \mathcal{R}_{1}, \mathcal{J} \mathcal{E R}_{2}\right) \leq 0$.

Now, let consider this three disjoint cases:

$$
\begin{aligned}
& \text { - } \mathcal{J E R}_{1}>\mathcal{J} \mathcal{E} \mathcal{R}_{2} \text { : } \\
& V_{1}{ }^{S E}-V_{1}{ }^{N E}=2\left(\frac{\alpha}{C}-N\right) / \mathcal{J E} \mathcal{R}_{1} \text {. } \\
& V_{2}{ }^{S E}-V_{2}{ }^{N E}=\left(\frac{\alpha}{C}-N\right) / \mathcal{J E} \mathcal{R}_{1} \text {. } \\
& \text { ○ } \mathcal{J E R}_{1}<\mathcal{J} \mathcal{E} \mathcal{R}_{2} \text { : } \\
& V_{1}{ }^{S E}-V_{1}{ }^{N E}=\left(\frac{\alpha}{C}-N\right) / \mathcal{J E} \mathcal{R}_{2} \text {. } \\
& V_{2}{ }^{S E}-V_{2}{ }^{N E}=2\left(\frac{\alpha}{C}-N\right) / \mathcal{J} \mathcal{E} \mathcal{R}_{2} \text {. } \\
& \text { ○ } \quad \mathcal{J} \mathcal{E} \mathcal{R}_{1}=\mathcal{J} \mathcal{E} \mathcal{R}_{2} \text { : } \\
& V_{1}{ }^{S E}-V_{1}{ }^{N E}=\left(\frac{\alpha}{C}+\beta_{1} J^{\prime}-N\right) / \mathcal{J E} \mathcal{R}_{1} \text {. } \\
& V_{2}{ }^{S E}-V_{2}{ }^{N E}=\left(2\left(\frac{\alpha}{C}-N\right)-\beta_{1} J^{\prime}\right) / \mathcal{J} \mathcal{E} \mathcal{R}_{1} \text {. } \\
& \text { where, } 0 \leq \beta_{1} J^{\prime} \leq(\alpha / C-N) \text {. }
\end{aligned}
$$

Thus $V_{i}^{S E}-V_{i}^{N E} \geq 0, \forall i \in\{1,2\}$.

- $R_{3}: \frac{\alpha}{C}>2 N$ :

$$
\begin{aligned}
& U^{S E}-U^{N E}= \\
& \left(\frac{\alpha}{4 C}\right) \cdot \max \left(\mathcal{J E} \mathcal{R}_{1}, \mathcal{J E} \mathcal{R}_{2}\right) \geq 0, P^{S E}-P^{N E}= \\
& \frac{-3 \alpha}{4 C^{2}} \cdot \max \left(\mathcal{J E} \mathcal{R}_{1}, \mathcal{J} \mathcal{E} \mathcal{R}_{2}\right) \leq 0 \text { and } S I N R^{S E}- \\
& S I N R^{N E}=\frac{-\alpha}{2 C} \cdot \max \left(\mathcal{J E} \mathcal{R}_{1}, \mathcal{J} \mathcal{E} \mathcal{R}_{2}\right) \leq 0 \text {. } \\
& \text { ○ } \mathcal{J E R}_{1}>\mathcal{J} \mathcal{E} \mathcal{R}_{2} \text { : } \\
& V_{1}{ }^{S E}-V_{1}{ }^{N E}=\frac{\alpha}{C} / \mathcal{J E} \mathcal{R}_{1} \text {. } \\
& V_{2}{ }^{S E}-V_{2}{ }^{N E}=\frac{\alpha}{2 C} / \mathcal{J E} \mathcal{R}_{1} \text {. } \\
& \text { ○ } \quad \mathcal{J E R}_{1}<\mathcal{J} \mathcal{E} \mathcal{R}_{2} \text { : } \\
& V_{1}{ }^{S E}-V_{1}{ }^{N E}=\frac{\alpha}{2 C} / \mathcal{J E} \mathcal{R}_{2} \text {. } \\
& V_{2}{ }^{S E}-V_{2}{ }^{N E}=\frac{\alpha}{C} / \mathcal{J E} \mathcal{R}_{2} \text {. } \\
& \text { ○ } \mathcal{J} \mathcal{E} \mathcal{R}_{1}=\mathcal{J} \mathcal{E} \mathcal{R}_{2} \text { : } \\
& V_{1}{ }^{S E}-V_{1}{ }^{N E}=\left(\frac{\alpha}{2 C}+\beta_{1}\left(J^{\prime}-J^{\prime \prime}\right)\right) / \mathcal{J E} \mathcal{R}_{1} \text {. } \\
& V_{2}{ }^{S E}-V_{2}{ }^{N E}=\left(\frac{\alpha}{C}+\beta_{1}\left(J^{\prime \prime}-J^{\prime}\right)\right) / \mathcal{J E} \mathcal{E} \mathcal{R}_{1} \text {. } \\
& \text { where, } 0 \leq \beta_{1} J^{\prime} \leq(\alpha / C-N) \text { and } 0 \leq \\
& \beta_{1} J^{\prime \prime} \leq(\alpha / 2 C-N) \text {. }
\end{aligned}
$$$$
\text { Thus } V_{i}{ }^{S E}-V_{i}{ }^{N E} \geq 0, \forall i \in\{1,2\} \text {. }
$$

\section{REFERENCES}

[1] H. Chen, C. Zhai, Y. Li and B. Vucetic, "Cooperative Strategies for Wireless-Powered Communications". arXiv preprint arXiv:1610.03527, 2016.

[2] M. Faub and AM. Zoubir, "Two Distributions Designed to Minimize the Expected Delay in CSMA Networks". IEEE Signal Processing Letters, , vol. 23, no. 2, p. 267-271, 2016.

[3] H. Zayandehroodi and M. Eslami, "Optimization of Energy Consumption in Cooperative Wireless Network using Quadratic Programming". Indian Journal of Science and Technology, vol. 8, no 35, 2015.

[4] RS. Cheng and CM. Huang, "Collision detect and avoidance media access mechanism for next generation 802.11 ax networks". In : Heterogeneous Networking for Quality, Reliability, Security and Robustness (QSHINE), 2015 11th International Conference on. IEEE p. 189-193, 2015.
[5] C. Kaufman and R. Perlman, M. Speciner, "Network security: private communication in a public world". Prentice Hall Press, 2002.

[6] M. Atallah, G. Kaddoum and L. Kong, "A survey on cooperative jamming applied to physical layer security". In : 2015 IEEE International Conference on Ubiquitous Wireless Broadband (ICUWB). IEEE. p. 1-5, October 2015

[7] Guan, Yanpeng, and Xiaohua Ge, "Distributed Attack Detection and Secure Estimation of Networked Cyber-Physical Systems Against False Data Injection Attacks and Jamming Attacks". IEEE Transactions on Signal and Information Processing over Networks, vol. 4, no. 1, p. 4859, March 2018.

[8] Pelechrinis, Konstantinos, Marios Iliofotou, and Srikanth V. Krishnamurthy. "Denial of service attacks in wireless networks: The case of jammers." IEEE Communications surveys and tutorials 13.2, p. 245-257, 2011.

[9] SD Amuru, HS Dhillon, RM Buehrer, "On Jamming Against wireless networks" IEEE Transactions on Wireless Communications, vol. 16, no. 1, pp. 412 - 428, November 2016.

[10] "IEEE standard for local and metropolitan area networks. part 16: Air interface for fixed and mobile broadband wireless access systems", february 2006.

[11] "IEEE standard for local and metropolitan area networks. part 16: Air interface for fixed broadband wireless access systems". April 2002.

[12] E. Altman, N. Bonneau, and M. Debbah, "Correlated equilibrium in access control for wireless communications". In : International Conference on Research in Networking. Springer Berlin Heidelberg, p. 173-183, May 2006.

[13] R. Gallager and D. Bertsekas, "Data Networks". Prentice Hall, Englewood Cliffs, New Jersey, 1987.

[14] E. Altman, K. Avrachenkov, R. Marquez and G. Miller, "Zero-sum constrained stochastic Games with independent state processes". Mathematical Methods of Operations Research, vol. 62, no. 3, p. 375-386, 2005.

[15] E. Altman, K. Avrachenkov and A. Garnaev, "A jamming Game in wireless networks with transmission Cost". In : International Conference on Network Control and Optimization. Springer Berlin Heidelberg. p. 1-12, June 2007.

[16] Y. Li, L. Xiao, J. Liu and Y. Tang "Power control stackelberg Game in cooperative anti-jamming communications". In Int. Conf. Game Theory for Networks (GameNETS), pp. 1-6, Nov. 2014.

[17] L. Xiao, Y. Li, J. Liu and Y. Zhao, "Power control with reinforcement learning in cooperative cognitive radio networks against jamming." The Journal of Supercomputing, vol. 71, no. 9, p. 3237-3257, 2015.

[18] D. Yang, J. Zhang, X. Fang and A. Richa "Optimal transmission power control in the presence of a smart jammer". In : Global Communications Conference (GLOBECOM), 2012 IEEE, p. 5506-5511,Dec 2012.

[19] L. Xiao, T. Chen, J. Liu and H. Dai "Anti-jamming Transmission Stackelberg Game with Observation Errors". IEEE Commun. Lett., vol.19, no.6 pp. 949-952, Jun. 2015.

[20] Moulay Abdellatif LMATER, Majed Haddad, Abdelillah Karouit and Abdelkrim Haqiq, "Smart Jamming Attacks in Wireless Networks During a Transmission Cycle: Stackelberg Game with Hierarchical Learning Solution" International Journal of Advanced Computer Science and Applications (IJACSA), 9(4), 2018

[21] L. Jia, F. Yao, Y. Sun, Y. Niu and Y. Zhu "Bayesian Stackelberg Game for Anti-jamming Transmission with Incomplete Information." IEEE Communications Letters, vol. 20, no. 10, p. 1991-1994, 2016.

[22] E. Altman, K. Avrachenkov and A. Garnaev, "Jamming in wireless networks: The case of several jammers". In : Game Theory for Networks, 2009. GameNets' 09. International Conference on. IEEE. p. 585-592, May 2009.

[23] L. Jia, et al., "A Hierarchical Learning Solution for Anti-Jamming Stackelberg Game With Discrete Power Strategies" IEEE Wireless Communications Letters, vol. 6, no. 6, pp. 818 - 821, August 2017. 